\title{
«DOING YOGA» \\ Zwischen neoliberaler und transformativer Selbstsorge
}

\author{
von MAJA FIGGE
}

1 Vgl. Farah Godrej: The Neoliberal Yogi and the Politics of Yoga, in: Political Theory, Jg. 45, Nr. 6, 2017, 1-29, hier 10, doi. org/10.1177/0090591716643604. 2 Vgl. Friedericke Hardering, Greta Wagner: Vom überforderten zum achtsamen Selbst? Zum Wandel der Subjektivität in der digitalen Arbeitswelt, in: Thomas Fuchs, Lukas Iwer, Stefano Micali (Hg.): Das überforderte Selbst. Zeitdiagnosen einer beschleunigten Gesellschaft, Berlin 2018, 258-275, hier 274.

3 Ebd., 275. Der globale Wirtschaftsfaktor Yoga wird hier aus Platzgründen nicht berücksichtigt.

4 Vgl. Michel Foucault: Die Ethik der Sorge um sich als Praxis der Freiheit (Gespräch mit Helmut Becker, Raul Fornet-Betancourt, Alfred Gomez-Müller, 20.1.1984), in: ders.: Schriften in vier Bänden, Bd. IV: 1980-1988, hg. v. Daniel Defert und François Ewald unter Mitarbeit von Jacques Lagrange, Frankfurt / M. 2005, 875-902, hier 886 .
Das Erste, was ich nach der pandemiebedingt verfrühten Rückkehr von meiner Forschungsreise in Indien in den ersten Lockdown-Wochen im März vergangenen Jahres gemacht habe, war, eine Flatrate bei meinem Yogastudio in der Nachbar_innenschaft zu buchen, das bereits nach wenigen Tagen auf ZoomUnterricht umgestellt hatte. Seither besuche ich wann immer möglich die Klassen, die eine Unterbrechung der ewigen Online-Videokonferenzen darstellen: Ohne Kamera, häufig mit geschlossenen Augen, folge ich der Stimme der Lehrerin, ihren Anleitungen, ihren Yoga-Floskeln, ${ }^{1}$ den philosophischen Erklärungen wie auch den Ermutigungen, und singe gemeinsam mit den anderen in Zoom Anwesenden Mantren in den Bildschirm meines Laptops. Sie helfen mir, eine Perspektive auf die Situation zu gewinnen, Abstand zu nehmen, Verbundenheit und Vertrauen zu spüren und meinem vom Home-Office schmerzenden und erschöpften Körper etwas Erholung zu verschaffen. Yoga ist hier Bewältigungsstrategie, um mit der Überforderung (der Online-Lehre und des social distancing) umzugehen. Es scheint einer neoliberalen Optimierungslogik verpflichtet, der die Vorstellung eines Subjekts zugrunde liegt, das seine Ressourcen schützt, eigene Prioritäten setzt und zunehmend <achtsamer〉 und damit fokussierter wird. ${ }^{2}$ Problematisch daran ist die Responsabilisierung des Individuums, weil diese die Fähigkeit zur kollektiven Kritik an den (Arbeits-) Bedingungen unterminiert. ${ }^{3}$ In Erweiterung dieser eindeutigen Einschätzung möchte ich dafür argumentieren, dass die in der Yogapraxis gewonnene Distanzierung auch eine Voraussetzung zur Analyse dieser Bedingungen sein kann, ebenso wie mit Michel Foucault die «Sorge um sich»Voraussetzung einer Sorge um andere und die Welt ist. ${ }^{4}$

Ich übe seit etwa I 5 Jahren Yoga, ungefähr zehn davon nach B.K. S. Iyengar, mit teils mehrjährigen Unterbrechungen der aktiven Praxis, aber aufgrund enger Beziehungen zu ausgebildeten Yogalehrer_innen in kontinuierlicher 
Auseinandersetzung darüber und damit. Ich würde behaupten, dass Yoga meine Haltung zum Leben verändert hat. Ich beziehe mich insbesondere auf die Konfrontation mit Scham und Angst sowie das Widerfahren von Glücksgefühlen im relativ geschützten Raum der Yogaklasse - und das über die Jahre erworbene (Körper-)Wissen, das mich die Verbundenheit der Welt lehrt und auf das ich überall und jederzeit zurückgreifen kann. Gleichzeitig scheint es mir notwendig, zwischen dem Üben von Yoga und dem Sprechen über Yoga zu unterscheiden. ${ }^{5}$ So vermag der Verweis auf die eigene Yogapraxis im Kolleg_innenkreis auch dazu dienen, das Bild zu vermitteln, dass man sich nicht gehen lässt, sondern etwas für die Gesundheit, die Selbstdisziplin und damit den eigenen Erfolg tut. Ein willkommener Nebeneffekt ist die Gemeinschaftsbildung mit anderen Übenden, die Behauptung eines Insiderwissens, das der Distinktion dient. Auch ich habe davon Gebrauch gemacht. Und so mag ich nicht ausschließen, dass die eingangs skizzierten Subjektivierungsprozesse nicht auch in meiner Yogapraxis am Werk sind.

Gerade die an Hochschulen angebotenen Klassen, die ich als Pendlerin ausprobierte, bestätigten begründete Annahmen über die Aneignungen von Yoga im Westen als ein vor allem von jungen, dünnen, <gesunden>, bildungsbürgerlichen weißen cis-Frauen praktiziertes Fitnessangebot. Meist waren die Anwesenden 15 bis 20 Jahre jünger als ich, mit Körpern in einer von mir nie erreichten Form, die nach Anleitung, aber ohne Aufwärmen, die Haltungen einnahmen, als wäre dies ein Kinderspiel, während ich mich entweder quälte oder aussetzte, aus Angst, mich zu verletzen. Diese body issues waren auch der Grund, warum ich mich für Iyengar-Yoga entschied und damit für einen Rückgriff auf alle möglichen Hilfsmittel: Decken, Kissen, Klötze, Gurte, Stühle, Säulen, Wände - Tools also ebenso wie Infrastrukturen. ${ }^{6}$ Unter Einbeziehung eines Wissens um die grundsätzliche Verletzlichkeit und Angewiesenheit sowie die Abwesenheit von <Gesundheit〉 wird der Körper - egal welcher Form, welchen Alters, welcher Kondition und Erkrankung - dabei unterstützt, zu üben. Außerdem mochte ich immer, dass wir zwar die Sanskrit-Namen der Asanas (āsana - Haltung) lernen, aber Yoga als etwas verstanden wird, dessen Genealogie zwar von Indien ausgeht, das jedoch als ein durch vielfältige Begegnungen verflochtenes, transkulturelles Phänomen beschrieben werden muss. So ist B.K.S. Iyengar einer der Gurus, die in der zweiten Hälfte des 20. Jahrhunderts zur großen Verbreitung von Yoga im Westen beigetragen haben. ${ }^{7}$ Mit der Geschichte und Philosophie von Yoga habe ich mich damals nicht weiter beschäftigt: ${ }^{8}$ vielleicht aus Abwehr des Verdachts kultureller Aneignung, ohne die eigene Verwicklung in dieses von post_kolonialen Machtbeziehungen durchzogene Gefüge - diesen double bind aus (neo-)kolonialen und (hindu-)nationalistischen Interessen und Auslegungen - ausreichend zu reflektieren. ${ }^{9}$

Gleichzeitig führte ich bereits zu Beginn meiner Beschäftigung mit Yoga Diskussionen darüber, ob Yoga nun neoliberale Selbstoptimierung sei oder eine solche gerade unterlaufe - festgemacht an der Frage des Übens, verstanden als
5 Verena Schnäbele arbeitet auf der Basis von Interviews mit Yogaübenden heraus, dass sich die Beschreibungen der Erfahrung ähneln. Betont werden die Auseinandersetzung mit körperlichen Grenzen sowie das Verhältnis zwischen innen und außen. Vgl. Verena Schnäbele: The Useful Body: The Yogic Answer to Appearance Management in the Post Fordist Workplace, in: Beatrix Hauser (Hg.): Traveling Yoga. Bodily Practice in Transcultural Perspective, Cham 2013, 135-153, hier $15^{2} \mathrm{f}$.

6 Vgl. Suzanne Newcombe: Spaces of Yoga: Towards a NonEssentialist Understanding of Yoga: Historical and Contemporary Perspectives, in: Karl Baier, Philipp A. Maas, Karin Preisendanz (Hg.): Yoga in Transformation, Göttingen 2018, 551-573.

7 Zur transnationalen Genealogie des modernen auf Asanas basierenden Yoga vgl. Beatrix Hauser: Introduction. Transcultural Yoga(s). Analyzing a Traveling Subject, in: dies.: Traveling Yoga, 1-34; Elizabeth de Michelis: A History of Modern Yoga. Patañjali and Western Esotericism, London 2004; Marc Singleton: Yoga Body. The Origins of Modern Posture Practice, Oxford 2010. Auch das anti$k e$, auf dem indischen Subkontinent verortete Yoga wird in der rezenten Forschung als transkulturell, heterogen und synkretistisch angesehen, vgl. Godrej: The Neoliberal Yogi, 3 .

8 Das moderne Yoga entstand im Dialog zwischen dem kolonialen Indien und den weltweiten Körperkulturbewegungen, die sich mit nationalistischen und antikolonialen Bestrebungen verbanden: «Indian yogis internalized the importance of physical fitness during the late colonial period, constructing indigenous> exercises partially borrowed from imported fitness techniques, resulting in a span-Indian hub of physical culture revivalism.) The methods of postural yoga that became popular in India in the early to mid-twentieth century swould not have been considered yoga prior to this period of Indian historys." Godrej: The Neoliberal Yogi, 4. 
9 Nach Shameem Black ist die Frage der kulturellen Aneignung «not a call for essentialism or exclusion, but instead an attempt to analyse the place of yoga within larger structures of material privilege, state power and dominant norms". Shameem Black: Decolonizing Yoga, in: Suzanne Newcombe, Karen O'Brien-Kop (Hg.): The Routledge Handbook of Yoga and Meditation Studies, Abingdon, New York 2021, 13-21, $16 \mathrm{f}$.

10 Godrej: The Neoliberal Yogi, 3.

11 Ebd.

12 vgl. ebd., 10.

13 Newcombe: Spaces of Yoga, 552.

14 Vgl. Schnäbele: The Useful Body, 153.

15 Vgl. einen Spiegel-Artikel, der versucht, westliche Überlegenheit zu generieren, indem er Yoga als Erfindung der westlichen Moderne deklariert. Ausgeblendet wird, dass diese nicht ausschließlich westlich und vor allem kolonial war: «Forscher enthüllen die wahre Herkunft der indischen Spiritualität: Die angeblich uralten Geisteslehren sind eine Erfindung der westlichen Moderne - und das gymnastische Yoga haben europäische Turner und Bodybuilder entwickelt.» Manfred Dworschak: Erlösung ohne Erlöser, in: Der Spiegel, Nr. 31, 2013, 96-101, hier 96.

16 Dies gilt auch für hindunationalistische Auslegungen von Yoga, vgl. Suzanne Newcombe: The Revival of Yoga in Contemporary India, in: John Barton (Hg.): Oxford Research Encyclopedias: Religion, Oxford 2017, doi.org/10.1093/ acre fore/9780199340378.013.253; Christopher Patrick Miller: Soft Power and Biopower: Narendra Modi's "Double Discourse» Concerning Yoga for Climate Change and SelfCare, in: Journal of Dharma Studies, Jg. 3, Nr. 1, 2020, 93-106.

17 Godrej: The Neoliberal Yogi, 2.

18 Vgl. ebd., 16-20.

19 Veena Howard: Gandhi's Ascetic Activism. Renunciation and Social Action, Albany 2013.
Einüben von Selbstdisziplin und Selbstbeherrschung (buchstäblich die Übersetzung des Begriffs $\left\langle\right.$ Yoga $>^{10}$ ), die auf das Erreichen eines veränderten Bewusstseinszustands, «usually defined as ultimate union with the divine», ${ }^{11}$ ausgerichtet ist. Zwar zielt die Praxis - im Iyengar-Yoga wie in vielen anderen Yogarichtungen - auf einen Fortschritt, auf Heilung, auf Selbst-Realisierung und kann damit durchaus im Sinne neoliberaler Selbsttechnologien interpretiert werden. ${ }^{12}$ Jedoch war es die wiederholende Einzigartigkeit jedes Übens, der Fokus auf das Jetzt, auf die eine Sache, den einen Atemzug oder den einzelnen Muskel - immer anders, jedes Mal neu -, gepaart mit der Anerkennung der eigenen, mit der Welt verbundenen Seele bei gleichzeitiger Zurücknahme des Egos, die mich - neben dem Spaß und der Bestärkung, die ich in Yogaklassen erfahren habe und weiter erfahre - fasziniert und zu der Einschätzung gebracht haben, dass Yoga auch ein Weg sein kann, den (selbst-)auferlegten neoliberalen Anforderungen zu entkommen und die Erfahrung des «doing Yoga ${ }^{13}$ für ein solidarisches In- und Mit-der-Welt-Sein zu öffnen, in Opposition zu Konkurrenz, Konsum, Erfolgsdruck, Selbstoptimierung und -vermarktung. Ein Anhaltspunkt dafür findet sich auch in der Yogaforschung, wenn etwa Verena Schnäbele auf Basis von Interviews zeigt, dass die Yoga-Übenden im Lauf ihrer Praxis der Differenz zwischen der durch Yoga erfahrenen Transformation und der gleich (prekär) bleibenden Arbeitsbedingungen zunehmend gewahr werden und in der Folge teilweise mikropolitische Widerstandspraktiken entwerfen. ${ }^{14}$

Vor diesem Hintergrund plädiere ich dafür, eine Ambivalenz zuzulassen: gegenüber den Behauptungen des <Authentischen> der uralten oder überzeitlichen indischen Yogakultur, gegenüber der (Ent-)Wendung, modernes Yoga sei eigentlich durch eine Aneignung europäischer Körpertechniken entstanden, ${ }^{15}$ aber auch gegenüber der teilweise reflexhaft vorgetragenen Verurteilung des neoliberalen Achtsamkeitshypes, die die Möglichkeiten des Widerstands aus dem Blick verliert. Dabei beziehe ich mich auf Farah Godrej, die versucht, genau diese Ambivalenz anzuerkennen. Godrej zeigt, dass Yoga zahlreiche Ressourcen und Anschlusspunkte bereitstellt, um die neoliberale Subjektivierung herauszufordern und Praktiken des Widerstands zu entwerfen. Gegen die teilweise in Nordamerika in linken aktivistischen Kontexten ${ }^{16}$ anzutreffende Behauptung einer <Authentizität> und <Ursprünglichkeit> von Yoga zeigt sie, dass erst eine spezifische Auslegung und Konstellation von Yogatexten und Praktiken die Möglichkeit birgt, Widerstand zu aktivieren. ${ }^{17}$ Godrej verortet diese Möglichkeit im sukzessiven Rückzug von weltlichem Streben nach Konsum, Erfolg, Besitz wie von der materiellen Welt (inklusive des Körpers), wie er etwa im Grundlagentext, Patañjalis Yōga-Sūtra, beschrieben wird. Das Potenzial einer transformativen Selbstsorge entfalte der Rückzug allerdings erst, wenn er, wie etwa in der Bhagavad-Gītā, mit einer weltlichen Ethik verbunden werde, dem karma yōga, als ethisch uneigennützigem Handeln zum Wohl aller. ${ }^{18}$ Godrej dient Mahatma Gandhis «Ascetic Activism», ${ }^{19}$ in welchem der Verzicht auf Bedürfnisse mit dem unermüdlichen 
Einsatz für politischen Wandel zusammengeht, als Beispiel für eine solche eklektische Kombination von Rückzug und selbstlosem, auf die Welt gerichtetem Handeln für einen yogischen politischen Aktivismus. Von der Bezugnahme auf und dem Praktizieren von Yoga in antikolonialen und postkolonialen Kontexten zieht sie eine Verbindungslinie zu aktuellen Praktiken, die das transformative und heilende Potenzial von Yoga in heutigen Kämpfen um soziale Gerechtigkeit einsetzen:

If contemporary yoga is taught in ways that keep it loosely yoked to these philosophical precepts, it contains the potential for a counterhegemonic construction of subjectivity, one that would stand in opposition to the norms of consumption, selfoptimization, self-investment, and depoliticization. ${ }^{20}$

Gegen komplexitätsreduzierende, vereindeutigende Lesarten von Yoga diese in der Theorie und selbstsorgenden Praxis liegenden transformativen und widerständigen Möglichkeiten stark zu machen (ohne existierende Machtverhältnisse aus den Augen zu verlieren), erscheint mir angesichts der ökonomischen und biopolitischen Indienstnahme von Yoga im Westen ebenso wie durch die derzeitige indische Regierung (auch während der Pandemie ${ }^{21}$ ) umso wichtiger. ${ }^{22}$
20 Vgl. Godrej: The Neoliberal Yogi, 20.

21 Vgl. Vidya Krishnan: Where Pseudoscience Is Spreading, in: The Atlantic, 18.8.2020, theatlantic.com/ international/archive/2020/08/amitabhbachchan-india-coronavirus|615310| (7.12.2020).

22 Wie das aussehen kann, lässt sich nachlesen in Beth Berila, Melanie Klein, Chelsea Jackson Roberts (Hg.): Yoga, the Body, and Embodied Social Change. An Intersectional Feminist Analysis, Lanham 2016, und im Online-Journal Race and Yoga (escholarship.org/uc/ crg_raceandyoga). 